\title{
Implementing COVID-19 Mitigation in the Community Mental Health Setting: March 2020 and Lessons Learned
}

\author{
Zakia Alavi $^{1,2}$ (D) Raza Haque $^{3} \cdot$ Isabella Theresa Felzer-Kim ${ }^{4} \cdot$ Todd Lewicki $^{1} \cdot$ Ali Haque $^{5} \cdot$ Meaghan Mormann $^{6}$
}

Received: 4 April 2020 / Accepted: 3 July 2020 / Published online: 17 July 2020

(c) Springer Science+Business Media, LLC, part of Springer Nature 2020

\begin{abstract}
In March 2020, at the beginning of the COVID-19 pandemic, state-funded community mental health service programs (CMHSP) in Michigan, organized into 10 regions known as a "Prepaid Inpatient Health Plan" (PIHP), grappled with the task of developing a modified plan of operations, while complying with mitigation and social distancing guidelines. With the premise that psychiatric care is essential healthcare, a panel of physician and non-physician leaders representing Region 5 , met and developed recommendations, and feedback iteratively, using an adaptive modified Delphi methodology. This facilitated the development of a service and patient prioritization document to triage and to deliver behavioral health services in 21 counties which comprised Region 5 PIHP. Our procedures were organized around the principles of mitigation and contingency management, like physical health service delivery paradigms. The purpose of this manuscript is to share region 5 PIHP's response; a process which has allowed continuity of care during these unprecedented times.
\end{abstract}

Keywords Community mental health · COVID-19 · COVID · Coronavirus · Psychiatry · Mental health · Behavioral health · Community mental health and covid-19 $\cdot$ Mental and physical comorbidities and covid-19 $\cdot$ Telehealth guidelines

\section{Introduction}

The novel Coronavirus 2019 (COVID-19) took health systems across the globe by surprise, leaving in its path significant emotional, social, and economic burdens. Federal and state level guidance, while much more detailed at this time, were modest and had predominantly originated from the Centers for Disease Control (CDC) and the Centers for

Electronic supplementary material The online version of this article (https://doi.org/10.1007/s10597-020-00677-6) contains supplementary material, which is available to authorized users.

Zakia Alavi

alavizak@msu.edu

Raza Haque

haque@msu.edu

Todd Lewicki

todd.lewicki@midstatehealthnetwork.org

Ali Haque

haqueal1@msu.edu

Meaghan Mormann

megmormann@gmail.com

1 Mid-State Health Network, Lansing, USA
Medicare \& Medicaid Services (CMS). Those guidelines were sweeping and broad, leaving communities, health systems, hospitals, nursing homes, and other points of care struggling to implement preventative measures while facing their own infrastructural and operational challenges (Gershman).

This crisis is affecting everyone, but those with pre-existing health conditions are most at risk of illness and hospitalization (Alavi et al. 2020; Daré et al. 2019; Groups at Higher Risk for Severe Illness 2020). Thus, certain segments of the population were particularly affected (Weiner 2020). These

2 Department of Pediatrics and Human Development, Michigan State University, Lansing, USA

3 Department of Family Medicine, Michigan State University, Lansing, USA

4 Department of Kinesiology, College of Education and College of Human Medicine, Michigan State University, Lansing, USA

5 PGY-1 Internal Medicine, Loyola University Medical Center, Hines, USA

6 PGY-1 Pediatrics, Northwestern University-Lurie Children's Hospital, Chicago, USA 
population subsets include adults with severe and persistent mental illness (SMI), children and adults with intellectual and/or developmental disabilities (IDD) (including autism), children with severe emotional disturbance (SED), and children and adults with substance use disorders (SUD) (Janssen et al. 2015).

As we have stated in another report: "Mental illnesses are common in the United States, with approximately 46.7 million persons (19.1\%) living with a mental illness, and 11.4 million (4.6\%) having serious/severe mental illness (SMI), meaning that they experience impairment that substantially interferes with or limits their life activities" (Alavi et al. 2020). In the United States, these populations frequently receive treatment through public-sector-managed behavioral health, also known as community mental health centers (CMHCs) or in Michigan, known as community mental health service programs (CMHSPs), which are grouped into Prepaid Inpatient Health Plans (PIHP) (Kohrt et al. 2018). PIHPs are entities identified in federal regulations by the CMS which provide behavioral and medical services to

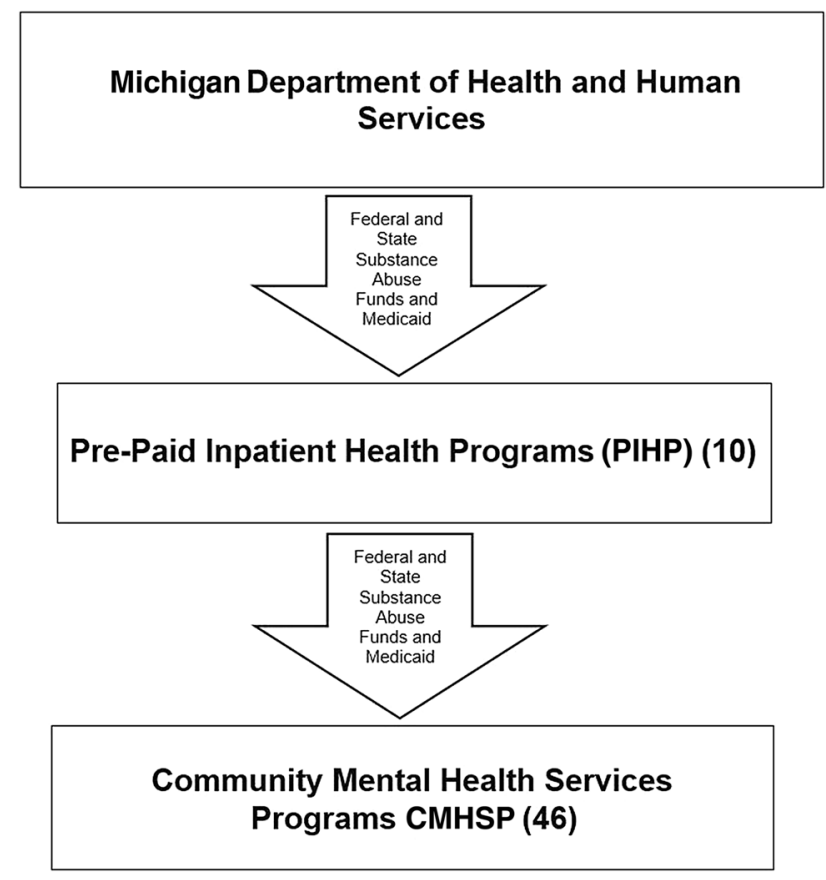

Fig. 1 Organizational structure of community mental health service providers (CMHSP) in Michigan eligible individuals including arranging psychiatric inpatient hospital care (What is a PIHP?. Community Mental Health Association of Michigan). There are 10 PIHPs in Michigan and their responsibility is to manage Medicaid resources for behavioral health, IDD, and SUD services for beneficiaries (Insert Fig. 1).

Each PIHP has a variable number of CMHSPs who provide behavioral health, IDD, and SUD services. CMSPs are the local, often county based providers that provide provide services such as substance use rehabilitation and treatment, care for persons with intellectual and developmental disabilities (IDD) to live independent lives (including congregate residential settings), care in the community for persons with SMI, services to families and children, and psychiatric care.

While all $\mathrm{CMH}$ service providers are mandated to have a general disaster plan, the scope of COVID-19 is unprecedented, rendering many of these plans insufficient (Holmes and Deb 2004). As the rate of COVID-19 infection grew in Michigan, a state of emergency was declared. Hospitals, ambulatory health care facilities (HCF) and all levels of health care delivery were asked to modify their operations in order to mitigate the spread of infection. At that time (March 2020), we could not find any specific guidelines regarding mitigation and service delivery alterations for behavioral health services generally, nor specifically for PIHPs or CMHCs. Below is the plan we formulated and immediately put into action. This plan was created to address the unique needs of our region (Region 5 PIHP). It is our hope that systems across the country can use these tools as a starting point or template for developing their own individualized protocols.

\section{Methods}

We began the needs assessment process in mid-March, almost immediately after the declaration of a state of emergency by Governor Gretchen Whitmer (2020). This process was initiated by the chief medical officer and chief behavioral health officer using a modified Delphi methodology, which is a group communication process involving obtaining the consensus opinion of a group of experts through conducting detailed examinations and discussions of a specific issue for the purposes of goal setting, policy investigation, or predicting the occurrence of future events 


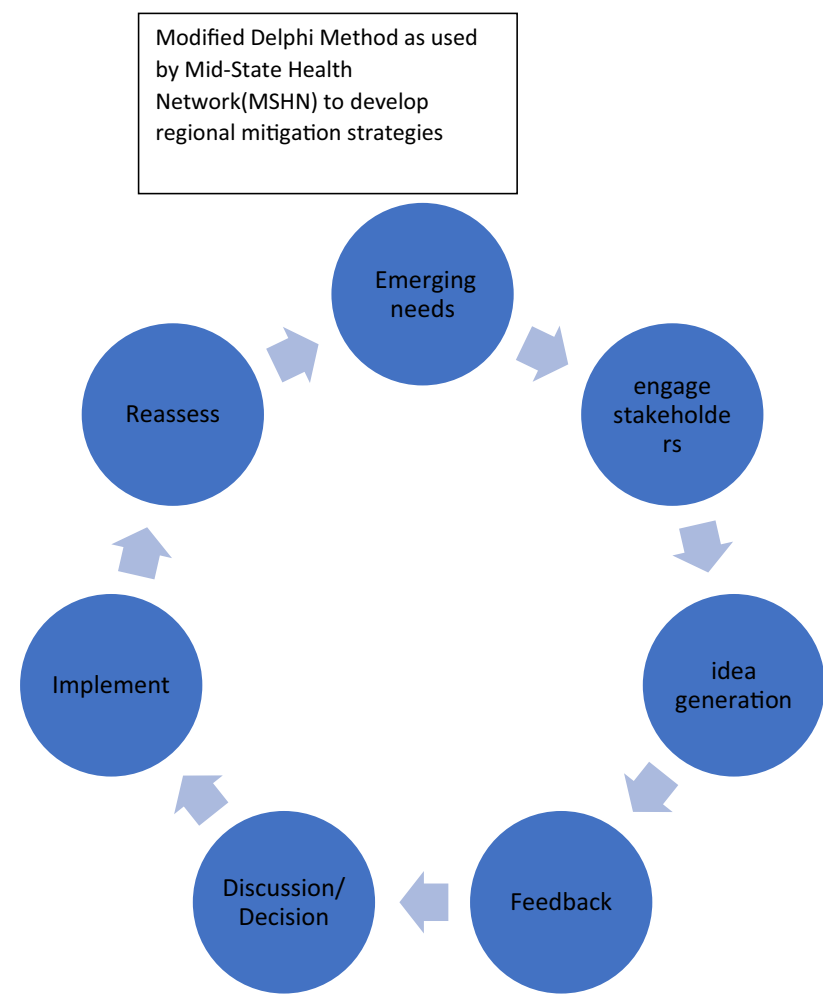

Fig. 2 Process for developing mitigation strategies based on Delphi Method

(Hasson and Keeney 2011; Hsu and Sandford 2007; Turoff and Hiltz 1996; Ludwig 1997). Our goal was to elicit opinions and expertise to ultimately achieve consensus on mitigation strategies for the administrative and clinical leadership of the 12 participating CMHSPs (Fig. 2) to apply in a standard fashion to their respective systems.

The group participated in multiple teleconference sessions of 2-3 h duration approximately 2-3 times per week, over a period of two weeks. The participants included medical directors of the Region 5 CMHSPs, their administrators, and their clinical directors. Our emergent needs were threefold: develop a unified mitigation strategy which could be standardized across the region served by the PIHP, develop and maintain clear lines of communication with timely and early physician input, and provide continuity of care across the various levels of care. We found these goals could be best addressed by targeting three areas: (i) communications, (ii) operations, and (iii) equipment.

The regional partners needed information to flow back from the CMHSPs to the PIHP seamlessly, reliably, and quickly. This was identified as a need in order to relay information regarding the CMHSP's regional COVID-19 count, disaster preparedness level, and personal protective equipment (PPE) needs back to the PIHP on a daily basis.

The other identified need was for unified, tier-based operations. We developed and implemented a modified 4-tier operating schedule that could be implemented across all counties while providing flexibility based on local disease spread. This was based on the CDC's guidelines and was modified to suit our needs (Document 1). As delineated above, a modified Delphi methodology was implemented to develop our consensus and the document was subsequently posted on the central PIHP website. As the pandemic progressed, this document became a central point of reference in communication between the PIHP and the CMHSPs

In our region, the most pertinent equipment needs were related to:

a) Information technology

b) Personal protective equipment (PPE)

Information technology equipment needs consisted primarily of telehealth encounter technology. Specifically, we needed to identify what platforms were best suited to the wide variety of services that are provided, test those platforms, and implement appropriate and practical formats for encounters. These were stratified by services that have both audio and visual components as opposed to telephone contact only.

PPE needs (similarly to most non-hospital-based healthcare personnel across the country) were largely surgical masks and N95 masks, gloves, goggles, gowns, and hand sanitizer. The situation was complicated by general unavailability of PPE-most behavioral health facilities do not stock and/or use PPE on a regular basis, and the sudden increased demand across the nation made it difficult to procure the needed quantities. Another PPE-related issue was training the staff in appropriate doffing and donning techniques; the staff at behavioral health facilities were unfamiliar with the principles of using PPE, unlike their counterparts in traditional physical healthcare facilities.

\section{Results}

Several processes and guiding documents were developed and implemented to continue operations, provide care to the Persons Served (PS), and optimize mitigation strategies.

\section{Processes}

At the administrative level, COVID19-specific weekly phone calls which included the participation of medical leadership were implemented. This level of communication was a major 
change for the PIHP, in which the network's Operations Council traditionally has limited contact with the chief medical officer, and has only quarterly contact with the medical directors of all CMHSPs.

A dedicated email account and resources webpage dedicated to COVID-19 were also created for the network and the local CMH. Additionally, we established a direct email/ text/phone link between the chief medical officer and the PIHP-designated senior staff member to allow for quick bidirectional communication. The next line of communication was opened between the chief medical officer and the medical directors of all of the member CMHSPs. This was set up as a weekly check-in phone call. Finally, each CMHC agreed to set up a specific email address and or phone line dedicated solely to fielding additional requests from patients for medication refill requests and other associated questions.

\section{Guiding Documents}

A 4-tier consensus protocol and statement regarding modified operations for the regional partners (see Document 1) was developed. All $12 \mathrm{CMH}$ programs agreed to report daily or more frequently as needed regarding active cases, emerging needs, and the tier/level of modified operations using a unified and standardized reporting grid provided to all CMHSPs (see Table 1 and Document 1). As the pandemic progressed, this document became a central point of reference in communication between the PIHP, the CMHSPs.

We reviewed the screening questionnaires that were developed by the CDC and the Health Department and combined them in a unified and standardized COVID-19 screening tool for all locations (see Document 2). Protocols for screening were established and standardized across all

Table 1 Reporting grid template for regional partner communication

\begin{tabular}{|l|l|}
\hline \multicolumn{2}{|c|}{ MSHN REGIONAL UPDATES } \\
\hline \multicolumn{2}{|c|}{ COVID-19 Reporting Template } \\
\hline $\begin{array}{l}|c| \\
\text { CMATUS UPDATE as of }\end{array}$ \\
\hline Active TIER & \\
\hline Consumer Count - Reported IIIness & \\
\hline Consumer Count - Confirmed & \\
\hline County(s) Affected & \\
\hline Supply/Demand Concerns: \\
\hline
\end{tabular}

regions. This included establishing a single point of entry to every facility and using the screening tool on every person entering each day. This also included self-screening for the front desk staff. Furthermore, for staff who had been in contact with a patient showing symptoms or screening positive, we required temperature checks every $6 \mathrm{~h}$ (Document 2 ).

Most face-to-face appointments, including psychiatry, psychotherapy, home-based care, and autism services were modified to occur by phone or via a virtual platform, unless it was absolutely necessary to see the patient in-person. The CMHSP partners asked for guidance regarding which patients were to be considered "absolutely necessary" to see in-person. In response to this request, we developed a document that provided guidance to medical and non-medical staff regarding scheduling patient appointments based on whether in-person contact was necessary. All routine appointments were transferred to remote care, including medication reviews, post-hospital visits with established and stable patients, and medication refills. We limited inperson visits to patients requiring injections, urgent psychiatric evaluations, or encounters regarding new and unstable conditions after hospitalization. Even for post-hospital encounters, we recommended first considering a telehealth encounter with the patient in a clinic and the physician on camera from a different site (Document 4, Table 2).

To assist all our providers who may be new to telehealth, we reviewed the American Psychiatric Association's telepsychiatry toolkit and ultimately decided that due to the unprecedented isolation of the required quarantine our needs were beyond the scope of these available guidelines (Toolkit and Association 2020). Therefore, we developed a short set of guidelines regarding the etiquette and framework of telehealth (phone or video) during pandemic times. We elected to base the framework on cognitive behavioral therapy and supportive psychotherapy as techniques which can be translated easily to a remote delivery format. In a pandemic situation, it was a concern that stigma, guilt, projection, splitting, and denial could be common, and guidelines reminded the provider to encourage reality testing as a coping mechanism (Ahmedani 2011). To reduce paranoia and obsessive thinking, the guidelines suggested providing the person served with a summary on how COVID-19 is and is not transmitted. To encourage self-directed action and empowerment, providers reiterated information on social distancing and hand washing techniques. The guidelines reminded providers to leave opportunities for questions regarding technology and accessibility. The guidelines for teletherapy are included in Document 3.

A list of risk factors in the CMHC population for COVID19-related complications was compiled based on the information available from the CDC and general medical wisdom. This list included older adults and individuals of any age with underlying medical issues such as asthma, chronic 
Table 2 Simplified contact precautions protocol for $\mathrm{CMH}$ staff on site

\begin{tabular}{|c|c|}
\hline Level of contact and relevant situations & Practices \\
\hline N95 masks & We are in a state of shortage \\
\hline $\begin{array}{l}\text { N95s are to be used only by: } \\
\text {-Actively symptomatic patient } \\
\text {-HCP in close contact with and caring for a patient who is symptomatic }\end{array}$ & $\begin{array}{l}\text { Limiting use to symptomatic patients or HCPs caring for them will } \\
\text { block the reservoir of infection }\end{array}$ \\
\hline Level 2 & Use gloves \\
\hline $\begin{array}{l}\text { Only when in close contact with: } \\
\text {-Anyone who has screened positive } \\
\text {-Anyone showing actual symptoms of respiratory infection }\end{array}$ & Use surgical masks and goggles \\
\hline Level 1 & Use standard precautions \\
\hline \multirow{2}{*}{$\begin{array}{l}\text { All screening staff and any HCP when in any proximity to: } \\
\text {-Any individual, even if they have screened negative or they have } \\
\text { unknown status }\end{array}$} & Erect a plastic barrier between staff and persons served \\
\hline & $\begin{array}{l}\text { If no barrier is available, use phones or tablets to communicate rather } \\
\text { than close contact and wear gloves while handling devices used by } \\
\text { the person served }\end{array}$ \\
\hline \multirow{3}{*}{$\begin{array}{l}\text { At all times } \\
\text { All staff always }\end{array}$} & Use a single point of entry for all individuals entering the facility \\
\hline & $\begin{array}{l}\text { Use a unified screener for all entering the facility } \\
\text { Screen everyone including yourself when you are onsite }\end{array}$ \\
\hline & Follow social distancing \\
\hline
\end{tabular}

$C M H$ community mental health; $H C P$ health care personnel; refers to all paid and unpaid persons serving in healthcare settings who have the potential for direct or indirect exposure to patients or infectious materials, including: body substances, contaminated medical supplies, devices, and equipment, contaminated environmental surfaces, contaminated air

Per CDC: "Based on what is currently known about COVID-19 and other coronaviruses, spread is thought to occur mostly from person-to-person via respiratory droplets among close contacts. Close contact can occur while caring for a patient, including:

-Being within approximately 6 feet $(2 \mathrm{~m})$ of a patient with COVID-19 for a prolonged period of time

-Having direct contact with infectious secretions from a patient with COVID-19. Infectious secretions may include sputum, serum, blood, and respiratory droplets

If close contact occurs while not wearing all recommended PPE, healthcare personnel may be at risk of infection"

For details see Document 4

Table 3 Stratification of patient encounters for remote care

\begin{tabular}{ll}
\hline $\begin{array}{l}\text { Routine appointments: CDC recommends remote } \\
\text { operations }\end{array}$ & $\begin{array}{l}\text { Routine medication reviews } \\
\text { Post-hospital visits when the patient is stable and is an existing patient } \\
\text { Medication refills }\end{array}$ \\
& Immunocompromised i.e. Clozaril group \\
& Patients taking medications for: \\
& Post-surgical care \\
& Diabetes \\
& Hypertension \\
& Other chronic condition: ie. MS, SLE, transplant recipients \\
& Patients taking oxygen \\
& Patients 70 or over \\
& Patients with asthma or COPD \\
Injections & Urgent Psychiatric evaluations recommended when a negative outcome is a risk in 30 days* \\
Post-hospital for a new and unstable patient (may be done by tele-med where the patient is & in the clinic and doctor is off-site on camera) \\
In-person appointments &
\end{tabular}

$C D C$ centers for disease control, $M S$ multiple sclerosis, $S L E$ systemic lupus erythematosus, $C O P D$ chronic obstructive pulmonary disease

*Negative outcomes generally seen as (a) inpatient treatment, (b) incarceration, (c) loss of life, or (d) acute or recent adverse drug reactions 
lung disease, serious heart conditions, severe obesity, etc. Also included were immunocompromised individuals such as those taking clozapine, those taking medications for diabetes mellitus, hypertension, post-surgical care, post-transplant care, or chronic conditions such as multiple sclerosis and systemic lupus erythematosus (Alavi et al. 2020; Daré et al. 2019; Groups at Higher Risk for Severe Illness 2020; CDC MMWR 2020; Manuel et al. 2013). Stratification guidelines are included in Table 3.

\section{Discussion}

Mid-state health network (MSHN) covers a large geographical area in mid-Michigan and is the Prepaid Inpatient Health Plan (PIHP) for twenty-one Michigan counties. Furthermore, MSHN operates in partnership with the CMHSPs agencies serving these counties. At the time of writing, our region along with the rest of the state is gradually beginning to emerge from the strictly enforced social distancing requirements. In the last several weeks, more clarity has emerged via the CDC and the State of Michigan regarding mitigation strategies and how they can be implemented in behavioral health settings. The processes and guiding documents that were developed have been helpful in providing a central point of reference and a unifying basis for maintaining operational integrity while mitigating risks associated with the pandemic. We recognize in many ways the process of needs assessment, discussion, consensus building, developing guidelines, implementing guidelines, and continuously reassessing guidelines was akin to building a plane while flying it. Although the lessons learned from our mitigation strategies are still emerging, we were able to successfully implement the infection control guidelines that were mandated by the CDC and Michigan Department of Health and Human Services (MDHHS). It is to be noted that the guidelines were not static, as new information emerged almost daily regarding the spread of infection and the level of mitigation needed. The operational communication was found by the team to be well directed and less frustrating than anticipated. The presence of a critical response team with operationalized physician involvement reduced the exposure of staff and kept the necessary services running properly by promptly making available communication and virtual visits. Our team leaders/physicians and managers reported increased efficiency in operational communication within the "chain of command" and increased clarity in caring for their patients during the pandemic. We were able implement a uniform screening and testing protocol to all mental health sites in 21 counties as per CDC guidelines by effectively identifying the structural needs of our organization and putting together an all inclusive chain of command and communication strategy with strong physician input and consistent policy implementation.

At the beginning of this process we anticipated challenges in the area of engagement from stakeholders and in implementation across a wide array of service delivery sites. We were pleasantly surprised to see that engagement from stakeholders, both administrative leadership and physician leaders, was enthusiastic and remained robust throughout the initial weeks and continues to be so at this time. The barriers that did emerge, and in some ways persisted, were related to resources and access to services that were beyond the reach of our region's capacity to address. These challenges included difficulty in finding psychiatric hospital beds for patients when they could not be maintained at home or in the community. Coordinating the mitigation efforts with the local health departments was another area requiring improvement because in Michigan, the state-funded local health departments and state-funded behavioral health services operate in parallel rather than in an integrated fashion. Another challenge was maintaining the quality of services that were delivered in the community such as services to individuals with autism, and intellectual and developmental disabilities. Providers of these services reported difficulty delivering services that have traditionally been provided with close personal contact between providers and persons served. Another unexpected challenge that emerged was related to manpower and availability of caregivers in settings where the risk of contracting COVID-19 was high. This was partially helped by increasing the reimbursement of direct care workers across the region; this was a financial undertaking that was substantial, occurred swiftly in response to an emerging need and mediated by frequent communication, and a uniform standard of operations across the 12 CMHSPs.

The guidelines outlined here are neither all-inclusive or universally prescriptive by any means. However, we feel they can serve as a template that could be adapted to any given point of care. The unique nature of CMHSPs, their ubiquitous distribution, and the diverse leadership in different communities necessitates their continued adaptation according to structural needs. Nonetheless, the readiness for disaster management in the CMHSP setting warrants discussion because of the vulnerable population receiving care. We hope that these resources are helpful in their preparation for any future communal emergencies and allows them to continue to protect vulnerable populations from such threats while safely providing optimal care during trying times and we hope to report the quantitative outcomes and projective modeling based on the impact of our mitigation strategies, as the pandemic becomes contained to further analyze the mental health system at different points of care. 


\section{Compliance with Ethical Standards}

Conflict of interest Authors have no conflicts of interest to declare.

\section{References}

Ahmedani, B. K. (2011). Mental health stigma: society, individuals, and the profession. The Journal of Social Work Values and Ethics, 8(2), 41-416.

Alavi, Z., Haque, R., Haque, A., \& Meier, D. (2020). COVID-19 pandemic mitigation for the high-risk patients served by the community mental health system. Consultant. https://doi.org/10.25270/ con.2020.05.00020.

CDC MMWR. (2020) Preliminary estimates of the prevalence of selected underlying health conditions among patients with coronavirus disease 2019-United States, February 12-March 28, 2020. Morbidity and Mortality Weekly Report. 69. https://doi. org/10.15585/mmwr.mm6913e2.

Daré, L. O., Bruand, P., Gérard, D., et al. (2019). Co-morbidities of mental disorders and chronic physical diseases in developing and emerging countries: A meta-analysis. BMC Public Health. https ://doi.org/10.1186/s12889-019-6623-6.

Gershman, J. A guide to state coronavirus lockdowns. Wall Street Journal. Retrieved April 3, 2020 from https://www.wsj.com/articles/astate-by-state-guide-to-coronavirus-lockdowns-11584749351.

Groups at Higher Risk for Severe Illness. Centers for Disease Control. (2020) Retrieved May 14, 2020 from https://www.cdc.gov/coron avirus/2019-ncov/need-extra-precautions/groups-at-higher-risk. html. Published 2020.

Hasson, F., \& Keeney, S. (2011). Enhancing rigor in the Delphi technique research. Technological Forecasting \& Social Change, 78, 1695-1704. https://doi.org/10.1016/j.techfore.2011.04.005.

Holmes, A. M., \& Deb, P. (2004). Performance assessment in community mental health care and at-risk populations. Health Care Financing Review, 26(1), 75-84.

Hsu, C. \& Sandford, B. (2007) The Delphi technique: Making sense of consensus. Practical Assessment, Research \& Evaluation. 12: 1-8. https://pareonline.net/pdf/v12n10.pdf.
Janssen, E. M., McGinty, E. E., Azrin, S. T., et al. (2015). (2015) Review of the evidence: Prevalence of medical conditions in the United States population with serious mental illness. General Hospital Psychiatry, 37(3), 199-222. https://doi.org/10.1016/j. genhosppsych.2015.03.004.

Kohrt, B. A., Asher, L., Bhardwaj, A., et al. (2018). The role of communities in mental health care in low- and middle-income countries: A meta-review of components and competencies. The International Journal of Environmental Research and Public Health, 15(6), 1279. https://doi.org/10.3390/ijerph15061279.

Ludwig, B. (1997) Predicting the future: Have you considered using the Delphi methodology? Tools of the Trade. 35(5).

Manuel, C. M., Rao, P. P., Rebello, P., et al. (2013). Medical comorbidity in in-patients with psychiatric disorder. Muller Journal of Medical Science and Research, 4, 12-17.

MSHN Guidance and Resources. https://midstatehealthnetwork.org/ provider-network-resources/provider-resources-1/coronaviru s-covid-19.

Telepsychiatry Toolkit. American Psychiatric Association. (2020) Retrieved April 3, 2020 from https://www.psychiatry.org/psych iatrists/practice/telepsychiatry/toolkit. Published 2020.

Turoff, M., \& Hiltz, S. (1996). Gazing into the Oracle: The Delphi technique and its application to social policy and public health. London, England: Jessica Kingsley Publishers.

Weiner, S. (2020). The new coronavirus affects us all, but some groups may suffer more. AAMC. https://www.aamc.org/news-insights/ new-coronavirus-affects-us-all-some-groups-may-suffer-more.

What is a PIHP?. Community Mental Health Association of Michigan. Retrieved April 3, 2020 from https://cmham.org/membership /pihp/.

Whitmer, G. (2020) Executive order 2020-21: Temporary requirement to suspend activities that are not necessary to sustain or protect life. Retrieved April 3, 2020 from https://www.xxxxxx.gov/whitm er/0,9309,7-387-90499_90705-522626--,00.html. Published March 24, 2020.

Publisher's Note Springer Nature remains neutral with regard to jurisdictional claims in published maps and institutional affiliations. 